\title{
NILPOTENCY INDICES OF THE RADICALS OF FINITE p-SOLVABLE GROUP ALGEBRAS, I
}

\author{
YASUSHI NINOMIYA
}

\section{Dedicated to Professor Yukio Tsushima on his 60th birthday}

(Received 10 May 2000; revised 6 December 2000)

Communicated by R. B. Howlett

\begin{abstract}
Let $k$ be a field of characteristic $p>0, G$ a finite $p$-solvable group and $p^{m}$ the highest power of $p$ dividing the order of $G$. We denote by $t(G)$ the nilpotency index of the (Jacobson) radical of the group algebra $k[G]$. The groups $G$ with $t(G) \geq p^{m-1}$ are already classified. The aim of this paper is to classify the $p$-solvable groups $G$ with $p^{m-2}<t(G)<p^{m-1}$ for $p$ odd.
\end{abstract}

2000 Mathematics subject classification: primary 20C05, $16 \mathrm{~S} 34$.

\section{Introduction}

Let $k$ be a field of characteristic $p>0$, and $G$ a finite group whose order is divisible by $p$. The (Jacobson) radical of the group algebra $k[G]$ will be denoted by $J(k[G])$. As it is well known, $J(k[G])$ is a nilpotent ideal. We denote by $t(G)$ its nilpotency index, that is, $t(G)$ is the least positive integer $t$ such that $J(k[G])^{t}=0$. Suppose that $G$ is $p$-solvable and let $p^{m}$ be the highest power of $p$ dividing the order of $G$. Then it is known that $t(G) \leq p^{m}$ (Passman, Tsushima, see [3, page 418]). We describe here the known results on the relation between the value of $t(G)$ and the structure of $G$. We first describe the known main results for the case of $p$-groups. In Theorem 1-Theorem 5 below, we assume that $G$ is a $p$-group of order $p^{m}$.

THEOREM 1 (Motose, Ninomiya [3, page 323]). $t(G)=p^{m}$ if and only if $G$ is cyclic.

(C) 2001 Australian Mathematical Society 0263-6115/2001 \$A2.00+0.00 
THEOREM 2 (Koshitani, Motose [3, page 325]). Let $m \geq 2$. Then $p^{m-1}<t(G)<p^{m}$ if and only if $\exp G=p^{m-1}$.

THEOREM 3 (Motose [3, page 326]). Let $m \geq 2$. Then $t(G)=p^{m-1}$ if and only if $G \cong M(3)$ or $G \cong C_{2} \times C_{2} \times C_{2}$.

THEOREM 4 (Ninomiya [6], Shalev [10]). Let $m \geq 3$. Then $p^{m-2}<t(G)<p^{m-1}$ if and only if one of the following holds:

(1) $p \neq 2, \exp G=p^{m-2}, G \not M(3)$.

(2) $p=5, m=4, G=\langle a, b, c, d\rangle: a^{5}=b^{5}=c^{5}=d^{5}=1,[c, d]=b$, $[b, d]=a$.

(3) $p=3, m=4, G \cong M(3) \times C_{3}$.

(4) $p=2, m \geq 4, \exp G=2^{m-2}$.

(5) $p=2, m=4, G \cong C_{2} \times C_{2} \times C_{2} \times C_{2}$.

(6) $p=2, m=5, G=\langle a, b, c\rangle: a^{2}=b^{4}=c^{4}=1,[b, c]=a$.

(7) $p=2, m=5, G=\langle a, b, c, d, e\rangle: a^{2}=b^{2}=c^{2}=d^{2}=1, e^{2}=c$, $[b, e]=[c, d]=a,[d, e]=b$.

In the presentation of the groups given in (2), (6) and (7), all relations of the form $[x, y]=1$ (with $x, y$ generators) are omitted .

THEOREM 5 (Ninomiya $[6,7])$. Let $m \geq 3$. Then $t(G)=p^{m-2}$ if and only if one of the following holds:

(1) $p=3, m=4, G \cong C_{3} \times C_{3} \times C_{3} \times C_{3}$.

(2) $p=3, m=5, G$ is nonabelian and $\exp G=3^{2}$.

(3) $p=2, m=5, \exp G=2^{2},|G / \Phi(G)|=2^{3}$.

We next describe the known main results for the case of $p$-solvable groups. In the rest of this section, $G$ will be assumed to be $p$-solvable, and $P$ a Sylow $p$-subgroup of $G$.

THEOREM 6 (Koshitani, Tsushima [3, page 419]). $t(G)=p^{m}$ if and only if $P$ is cyclic.

It is known that the groups $G$ of $p$-length 1 satisfy the equality $t(G)=t(P)$ (see [3, page 418]), and hence if $G$ has $p$-length 1 and its Sylow $p$-subgroups are of exponent $p^{m-1}$, then $p^{m-1}<t(G)<p^{m}$ by Theorem 2. On the other hand, for $p$-solvable groups of $p$-length greater than 1 , the following holds:

THEOREM 7 (Koshitani, Motose [3, page 440]). Let $\Phi / O_{p^{\prime}}(G)$ be the Frattini subgroup of $O_{p^{\prime}, p}(G) / O_{p^{\prime}}(G)$. Assume that $G$ is not of $p$-length 1 and $t(G)>p^{m-1}$. Then $p=2, G / \Phi \cong S_{4}$ and $\Phi / O_{p^{\prime}}(G)$ is cyclic. 
Let $p=2$. Motose showed that among the groups with the property $G / \Phi \cong S_{4}$ and $\Phi / O_{2^{\prime}}(G) \cong C_{2}$ there exist two types of groups: one of them satisfies $t(G)=9>8$, and the other satisfies $t(G)=7<8$ (see [3, page 445]). This shows that it is difficult to classify the groups with $2^{m-1}<t(G)<2^{m}$ and Theorem 2 cannot be extended to arbitrary $p$-solvable groups. But, for the case $p$ odd, Theorem 2 can be extended to $p$-solvable groups.

THEOREM 8 (Koshitani [3, page 442]). Let $p \neq 2$. Then the following are equivalent:

(1) $p^{m-1}<t(G)<p^{m}$.

(2) $t(G)=p^{m-1}+p-1$.

(3) $\exp P=p^{m-1}$.

Further, in this case, G has p-length 1.

We now suppose $t(G)=p^{m-1}$. If $G$ has $p$-length 1 then, because $t(G)=t(P)$, it holds by Theorem 3 that $P \cong M(3)$ or $P \cong C_{2} \times C_{2} \times C_{2}$. The following shows that the converse holds when $p \neq 2$ :

THEOREM 9 (Motose [3, page 449]). Let $p \neq 2$. Then $t(G)=p^{m-1}$ if and only if $p=3$ and $P \cong M(3)$.

The aim of this paper is to classify the $p$-solvable groups $G$ with $p^{m-2}<t(G)<$ $p^{m-1}$ for $p$ odd. Suppose $p^{m-2}<t(G)<p^{m-1}$. If $G$ has $p$-length 1 , then $P$ is one of the groups described in Theorem 4 . One of our results given below shows that the converse holds when $p \geq 5$.

THEOREM 10. Let $p \geq 5$. Then $p^{m-2}<t(G)<p^{m-1}$ if and only if $\exp P=p^{m-2}$ or $P$ is isomorphic to the group given in Theorem 4 (2). Further, in this case, $G$ has p-length 1 .

Our result for the case $p=3$ is as follows:

THEOREM 11. Let $p=3$ and $m \geq 3$. Suppose $3^{m-2}<t(G)<3^{m-1}$. If the 3-length of $G$ is greater than 1, then $G$ has 3-length 2. Suppose further that $O_{3^{\prime}}(G)=1$. Then $H=O_{3,3}{ }^{3},(G)$ is one of the groups from the following list:

(1) a nonsplit extension of

$$
\left\langle a, b, c \mid a^{3^{m-3}}=b^{3}=c^{3}=1,[a, b]=1,[a, c]=1,[b, c]=a^{3^{m-4}}\right\rangle
$$

by $\operatorname{SL}(2,3)(m \geq 5)$;

(2) a split extension of $C_{9} \times C_{9}$ by $\operatorname{SL}(2,3)$;

(3) an extension of $M(3)$ by $S L(2,3)$; 
(4) an extension of $C_{3} \times C_{3} \times C_{3}$ by $\mathrm{SL}(2,3)$;

(5) a split extension of $C_{3} \times C_{3} \times C_{3}$ by $A_{4}$; and

(6) a nonsplit extension of $C_{3^{m-3}} \times C_{3} \times C_{3}$ by $\mathrm{SL}(2,3)(m \geq 5)$.

As it is well known, the principal block (ideal) $B_{0}$ of $k[G]$ is isomorphic to $k\left[G / O_{p}^{\prime}(G)\right]$ (see [3, page 115]), and so Theorem 10 and Theorem 11 give a classification of $p$-solvable groups $G, p$ odd, with $p^{m-2}<t\left(B_{0}\right)<p^{m-1}$, where $t\left(B_{0}\right)$ is the nilpotency index of the radical of $B_{0}$. Further, we see that if $p^{m-2}<t\left(B_{0}\right)<p^{m-1}$, then it holds that $p^{m-2}<t(G)<p^{m-1}$.

In Section 2, we shall give the proof of Theorem 10 and the structure of $O_{3^{\prime}, 3}(G) / O_{3^{\prime}}(G)$ for 3-solvable groups $G$ satisfying the inequality $3^{m-2}<t(G)<3^{m-1}$. In Section 3, we shall give an outline of the proof of Theorem 11.

Notation is as follows:

$C_{n} \quad$ the cyclic group of order $n$

$Q_{8} \quad$ the quaternion group of order 8

$M$ (3) the exstra-special 3-group of order $3^{3}$ and exponent 3

$A_{4} \quad$ the alternating group on four letters

$\operatorname{SL}(n, p) \quad$ the special linear group of degree $n$ over the field of $p$ elements

$\mathrm{GL}(n, p)$ the general linear group of degree $n$ over the field of $p$ elements

$\Phi(G) \quad$ the Frattini subgroup of $G$

$\exp G \quad$ the exponent of $G$

$K: H \quad$ the semidirect product of $K$ by $H$

\section{Preliminaries}

Let $G$ be a $p$-solvable group satisfying $p^{m-2}<t(G)<p^{m-1}$. Assume now that the $p$-length of $G$ is greater than 1 and set $M=O_{p^{\prime}}(G), N=O_{p^{\prime}, p}(G)$ and $p^{r}=|N / M|$. Then, because $J(k[G])^{p^{m-r}} \subset J(k[N]) k[G]$ (Passman [3, page 110]), we have

$$
0 \neq J(k[G])^{p^{m-2}}=\left(J(k[G])^{p^{m-r}}\right)^{p^{r-2}} \subset J(k[N])^{p^{r-2}} k[G] .
$$

Thus we have $J(k[N])^{p^{r-2}} \neq 0$, that is, $t(N)>p^{r-2}$. Hence

$$
t(N / M)=t(N)>p^{r-2} .
$$

Let $\Phi / M$ be the Frattini subgroup of $N / M$. Since $G / N$ is contained isomorphically in Aut $N / \Phi$ (Hall and Higman [3, page 415]), $N / \Phi$ is not cyclic. Hence $N / M$ is not cyclic, and so by Theorem 1, $t(N / M)<p^{r}$. We therefore have

$$
p^{r-2}<t(N / M)<p^{r} .
$$

Hence, Theorem 2, Theorem 3 and Theorem 4 apply to the group $N / M$. Thus we have the following: 
LEMMA 1. We have the following possibilities:

(1) If $p^{r-1}<t(N / M)<p^{r}$ then $\exp N / M=p^{r-1}$.

(2) If $t(N / M)=p^{r-1}$ then, $N / M \cong M(3)$ or $C_{2} \times C_{2} \times C_{2}$.

(3) If $p^{r-2}<t(N / M)<p^{r-1}$, then $N / M$ is one of the groups given in Theorem 4 .

We first consider the case when $p \geq 5$ and prove Theorem 10 .

PROOF OF THEOREM 10. Assume that $p^{m-2}<t(G)<p^{m-1}$ and the $p$-length of $G$ is greater than 1 . Because $p \geq 5$, Lemma 1 implies that $N / \Phi \cong C_{p} \times C_{p}$ or $C_{p} \times C_{p} \times C_{p}$. Hence we may regard the group $G / N$ as a subgroup of $\operatorname{GL}(2, p)$ or $\mathrm{GL}(3, p)$. Then we see that every $p$-element of $G / N$ is of order $p$, and conjugate to an element given as follows:

$$
\left(\begin{array}{ll}
1 & 0 \\
\lambda & 1
\end{array}\right) \text { or }\left(\begin{array}{lll}
1 & 0 & 0 \\
\lambda & 1 & 0 \\
\nu & \mu & 1
\end{array}\right) \quad(\lambda, \mu, v \in \mathrm{GF}(p))
$$

Because the minimal polynomials of these matrices are $(X-1)^{2}$ and $(X-1)^{3}$ respectively, by Hall-Higman's Theorem B [2], we have $p=2$ or 3 . This contradicts our choice of $p$. Thus $G$ has $p$-length 1 and hence $t(G)=t(P)$. Therefore, by Theorem 4, $P$ is of exponent $p^{m-2}$ or isomorphic to the 5-group given in Theorem 4 (2).

To complete the proof of the theorem, it suffices to prove that if $p \geq 5$ and $P$ is of exponent $p^{m-2}$ or isomorphic to the 5-group given in Theorem 4 (2), then $G$ has $p$-length 1. It is well known that $G$ has $p$-length 1 provided that $P$ is abelian. Suppose now that $P$ is nonabelian. If $\exp P=p^{m-2}$, then $P$ is one of the groups $G_{i}$ $(i=1, \ldots, 10)$ given in [5, Theorem 1]. Each of these groups is of class at most 3, and the 5-group given in Theorem $4(2)$ is of class 3 . Hence $P$ is of class at most 3 in either case. Then by [2, Theorem 3.4.1], $G$ has $p$-length 1 . Thus the result follows.

In the case when $p=2$, as it is stated in Section 1, even the classification of the groups satisfying $2^{m-1} \leq(G)<2^{m}$ cannot be accomplished yet. So it is hard to classify the groups satisfying $2^{m-2}<t(G)<2^{m-1}$. Hence we restrict our attension to the case of $p$ odd. By Theorem 10, we are done when $p \geq 5$. Hence, in what follows, we consider the case $p=3$ only. We already know that if $G$ has 3-length 1, then $3^{m-2}<t(G)<3^{m-1}$ if and only if $\exp P=3^{m-2}$ with the exception of $M(3)$ or $P \cong M(3) \times C_{3}$. Suppose now that the 3-length of $G$ is greater than 1. Because the 3-group $N / M$ saisfies the inequality $3^{r-2}<t(N / M)<3^{r}, N / M$ is one of the following groups:

$$
C_{3^{\cdots}+} \times C_{3}, \quad M_{r}(3), \quad \exp N / M=3^{r-2}, \quad M(3) \times C_{3},
$$


where $M_{r}(3)$ is a nonabelian 3-group of order $3^{r}$ and exponent $3^{r-1}$. This implies that $N / M$ is generated by two or three elements, and so $G / N$ is contained isomorphically in $\operatorname{GL}(2,3)$ or $\operatorname{GL}(3,3)$. Hence noting that $\operatorname{GL}(2,3)=\left(Q_{8}: C_{3}\right): C_{2}$ and the maximal subgroups of $\operatorname{SL}(3,3)$ are $S_{4}, C_{13}: C_{3}$ and $\left(C_{3} \times C_{3}\right): H$ with $H / C_{2} \cong S_{4}$ (see [1]), we have the following:

LEMMA 2. The 3-part of $|G / N|$ is 3, and if $G / N$ is of even order and $N / M$ is generated by exactly two elements, then $|G / N|$ is divisible by 8 .

Since $C_{G}(N / M) \subset N$ (Hall and Higman [3, page 415]), we see that $G / N$ is isomorphic to a quotient group of some subgroup of Aut $N / M$. We give here the order of the automorphism groups of 3-groups given above:

$$
\begin{gathered}
\mid \text { Aut } C_{3^{r-1}} \times C_{3} \mid= \begin{cases}2^{4} \cdot 3 & \text { if } r=2 ; \\
2^{2} \cdot 3^{r} & \text { if } r>2,\end{cases} \\
\mid \text { Aut } M_{r}(3)\left|=2 \cdot 3^{r}, \quad\right| \text { Aut } M(3) \times C_{3} \mid=2^{5} \cdot 3^{6} .
\end{gathered}
$$

Assume next that the 3-group is of exponent $3^{r-2}$. If it is abelian, then it is either $C_{3^{\prime-2}} \times C_{3^{2}}(r \geq 4)$ or $C_{3^{r-2}} \times C_{3} \times C_{3}(r \geq 3)$, and we have

$$
\begin{array}{r}
\text { Aut } C_{3^{r-2}} \times C_{33^{2}} \mid= \begin{cases}2^{4} \cdot 3^{5} & \text { if } r=4 ; \\
2^{2} \cdot 3^{r+2} & \text { if } r>4,\end{cases} \\
\text { Aut } C_{3^{r-2}} \times C_{3} \times C_{3} \mid= \begin{cases}2^{5} \cdot 3^{3} \cdot 13 & \text { if } r=3 ; \\
2^{5} \cdot 3^{r+2} & \text { if } r>3\end{cases}
\end{array}
$$

If the 3-group is nonabelian, it is one of the groups $G_{1}, \ldots, G_{11}$ given in [5, Theorem 1], and we have

$$
\mid \text { Aut } G_{1} \mid=\left\{\begin{array}{ll}
2^{4} \cdot 3^{3} & \text { if } r=3 ; \\
2^{2} \cdot 3^{r+1} & \text { if } r>3,
\end{array} \quad \text { Aut } G_{2} \mid= \begin{cases}2 \cdot 3^{5} & \text { if } r=4 ; \\
2 \cdot 3^{r+2} & \text { if } r>4,\end{cases}\right.
$$

$\mid$ Aut $G_{3}\left|=2^{2} \cdot 3^{r+2}, \quad\right|$ Aut $G_{4}\left|=2^{4} \cdot 3^{r} . \quad\right|$ Aut $G_{5}\left|=2^{2} \cdot 3^{r}, \quad\right|$ Aut $G_{6} \mid=2^{2} \cdot 3^{r}$

$$
\begin{gathered}
\mid \text { Aut } G_{7} \mid=\left\{\begin{array}{ll}
2 \cdot 3^{4} & \text { if } r=4 ; \\
2 \cdot 3^{r+1} & \text { if } r>4,
\end{array} \quad \mid \text { Aut } G_{8} \mid= \begin{cases}2 \cdot 3^{5} & \text { if } r=5 ; \\
2 \cdot 3^{r+1} & \text { if } r>5,\end{cases} \right. \\
\mid \text { Aut } G_{9}\left|=2 \cdot 3^{r+1}, \quad\right| \text { Aut } G_{10}\left|=3^{r+1}, \quad\right| \text { Aut } G_{1 !} \mid=2 \cdot 3^{5} .
\end{gathered}
$$

Because $G_{1} \cong M(3)$, if $r=3$ and Aut $G_{3}$ is 3-closed, the above together with Lemma 2 implies that the possibility for $N / M$ is as follows:

$$
C_{3} \times C_{3}, \quad C_{3^{m-3}} \times C_{3} \times C_{3}, \quad G_{4}, \quad C_{9} \times C_{9}, \quad M(3) \times C_{3}, \quad M(3) .
$$


To prove Theorem 11, we need to calculate the value of $t(G)$ for 3-solvable groups $G$ of 3-length greater than 1. But, in general, it is difficult to determine $J(k[G])$ explicitly for $p$-solvable groups $G$ of $p$-length greater than 1 . In this context, Motose has described $J(k[Q d(3)])$ concretely and found its nilpotency index, where $Q d(3)$ is a semidirect product $\left(C_{3} \times C_{3}\right): \operatorname{SL}(2,3)$ with respect to the natural action of $\operatorname{SL}(2,3)$ on $C_{3} \times C_{3}$. This plays an important role in our proof. We here describe his result. In what follows, we use the following notation:

$$
\begin{aligned}
X^{+} & =\sum_{x \in X} x, \text { where } X \text { is a finite subset of } k[G], \\
g^{+} & =\sum_{x \in\{g\}} x, \text { where } g \in G .
\end{aligned}
$$

Assume $p=3$. Let $Q=\langle x, y\rangle\left(\cong Q_{8}\right)$ be a Sylow 2-subgroup of $Q d(3)$ and $\sigma$ an element of $Q d(3)-O_{3}(Q d(3))$ of order 3. Moreover, we set $T=\left\{f, \tau, \tau^{2}\right\}$, where $f=x^{2}-1$ and $\tau=\sigma(1+x+y-x y) f$. Then $T$ is a 3-group with identity $f$ of order 3 . We now choose three nilpotent right ideals of $k[Q d(3)]$ :

$$
\begin{gathered}
A=J(k[\langle\sigma\rangle]) Q^{+} k[Q d(3)], \quad B=J(k[T]) k[Q d(3)], \\
C=J\left(k\left[O_{3}(\operatorname{Qd}(3))\right]\right) k[Q d(3)] .
\end{gathered}
$$

Motose's result is as follows:

Lemma 3 (Motose [3, Chapter 7, Section 4]). Let $p=3$. Then the following holds:

(1) $J(k[Q d(3)])=A+B+C$.

(2) $(A+B)^{5}=0$.

(3) $t(\operatorname{Qd}(3))=9$.

This also implies that if $3^{m-2}<t(G)<3^{m-1}$, then $N / M$ is not isomorphic to $C_{3} \times C_{3}$. Thus we have the following:

LEMMA 4. Let $m \geq 3$. Assume that the 3-length of $G$ is greater than 1 . If $3^{m-2}<t(G)<3^{m-1}$, then the possibilities for $N / M$ are as follows:

$$
C_{3^{m-3}} \times C_{3} \times C_{3}, \quad G_{4}, \quad C_{9} \times C_{9}, \quad M(3) \times C_{3}, \quad M(3) .
$$

\section{Outline of the proof of Theorem 11}

In this section, we shall give an outline of the proof of Theorem 11. In what follows, we assume that $p=3$ and $G$ is a 3 -solvable group of 3-length greater than 1 which 
satisfies the inequality $3^{m-2}<t(G)<3^{m-1}$. Then by Lemma $2, G$ has 3-length 2 . We now suppose that $M=O_{3^{\prime}}(G)=1$ and set $N=O_{3}(G)$. Because $J(k[G])=$ $J\left(k\left[O_{3,3^{\prime}, 3}(G)\right]\right) k[G]$ (Villamayor [3, page 108]), we have $t(G)=t\left(O_{3,3^{\prime}, 3}(G)\right.$ ). We may therefore assume that $G=O_{3,3^{\prime}, 3}(G)$. Then by Lemma 4 , it suffices to prove that

(i) if $N \cong G_{4}$ then (1) holds;

(ii) if $N \cong C_{9} \times C_{9}$ then (2) holds;

(iii) if $N \cong M(3)$ then (3) holds;

(iv) if $N \cong C_{3} \times C_{3} \times C_{3}$ then (4) or (5) holds;

(v) if $N \cong C_{3^{m-3}} \times C_{3} \times C_{3}$ with $m \geq 5$ then (6) holds, and

(vi) the case " $N \cong M(3) \times C_{3}$ " does not occur.

We begin with a proof of (i):

LEMMA 5. If $N \cong G_{4}$, then (1) holds.

PROOF. Suppose that $N$ is a group of order $p^{r}$ isomorphic to $G_{4}$, where $p$ is any odd prime. Then $N$ has a presentation:

$$
N=\left\langle a, b, c \mid a^{p^{r-2}}=b^{p}=c^{p}=1,[a, b]=1,[a, c]=1,[b, c]=a^{p^{r-3}}\right\rangle .
$$

We see that any automorphism $\phi$ of $N$ is given by

$$
\phi(a)=a^{i}, \quad \phi(b)=a^{p^{r-3} \alpha} b^{\beta} c^{\gamma}, \quad \phi(c)=a^{p^{m-3} x} b^{y} c^{z},
$$

where $(i, p)=1, i \equiv \beta z-\gamma y(\bmod p)$. Thus we have $\mid$ Aut $N \mid=p^{r}(p+1)(p-1)^{2}$. Evidently, any automorphism $\phi$ of $N$ induces an automorphism of $N / \Phi(N)$. We denote this by $\bar{\phi}$. Since $\Phi(N)=\left\langle a^{p}\right\rangle, \bar{\phi}=\overline{1}$ if and only if $i \equiv 1(\bmod p)$ and $\beta=z=1, \gamma=y=0$. This shows that $K=\{\phi \in$ Aut $N \mid \bar{\phi}=\overline{1}\}$ is a normal subgroup of Aut $N$ of order $p^{r-1}$.

Suppose now $p=3$. Then $\mid$ Aut $N \mid=2^{4} \cdot 3^{r}$ and $\mid$ Aut $N: K \mid=2^{4} \cdot 3$. We now choose three elements $\varphi, \psi, \eta$ of Aut $N$ given by

$$
\begin{array}{lll}
\varphi: a \rightarrow a, & b \rightarrow b^{2}, & c \rightarrow b, \\
\psi: a \rightarrow a, & b \rightarrow a^{2 \cdot 3^{r-3}} b c^{2}, & c \rightarrow a^{3^{r-3}} b^{2} c^{2}, \\
\eta: a \rightarrow a, & b \rightarrow b, & c \rightarrow a^{3^{r-3}} b c .
\end{array}
$$

Then $\langle\varphi, \psi\rangle \cong Q_{8}, \eta^{3}=1$, and $\eta$ acts on $\langle\varphi, \psi\rangle$ as follows:

$$
\eta^{-1} \varphi \eta=\varphi^{3} \psi, \quad \eta^{-1} \psi \eta=\varphi .
$$

We therefore see that $K:\langle\varphi, \psi, \eta\rangle$ is a (normal) subgroup of Aut $N$ of index 2. Because $O_{3,3^{\prime}, 3}(G)=G$, this shows that $G / N \cong \operatorname{SL}(2,3)$. We now let $x, y, \sigma$ be the 
elements of $G$ corresponding to $\varphi, \psi, \eta$ respectively. Then $\langle x, y\rangle \cong Q_{8}, \sigma^{3} \in N$ and $\sigma^{-1} x \sigma=x^{3} y, \sigma^{-1} y \sigma=x$. Since $\sigma^{3} \in Z(N)=\langle a\rangle$ and $[a, \sigma]=1,\langle a, \sigma\rangle$ is either cyclic or isomorphic to $C_{3^{r-2}} \times C_{3}$. To prove the lemma, it suffices to show that if the former case holds then $3^{m-2}<t(G)<3^{m-1}$ and if the latter case holds then $t(G)<3^{m-2}$.

Suppose first $\langle a, \sigma\rangle$ is cyclic. Then we have

$$
G /\langle a\rangle \cong Q d(3) \text { and } k[G] / J(k[\langle a\rangle]) k[G] \cong k[G /\langle a\rangle] .
$$

Hence, by Lemma 3 ,

$$
Q^{+} J(k[\langle\sigma\rangle]) \subset J(k[G])
$$

where $Q=\langle x, y\rangle$. Since

$$
\left(Q^{+} J(k[\langle\sigma\rangle])\right)^{3^{r-1}-1}=k Q^{+} \sigma^{+},
$$

noting that $b-1 \in J(k[N]) \subset J(k[G])$, we have

$$
J(k[G])^{3^{m-2}}=J(k[G])^{3^{r-1}} \supset\left(Q^{+} J(k[\langle\sigma\rangle])\right)^{3^{r-1}-1}(b-1)=k Q^{+} \sigma^{+}(b-1) \neq 0 .
$$

Thus we have $t(G)>3^{m-2}$, and so $3^{m-2}<t(G)<3^{m-1}$ by Theorem 6 , Theorem 8 and Theorem 9.

We assume next that $\langle a, \sigma) \cong C_{3^{r-2}} \times C_{3}$. Then we may assume that $\sigma^{3}=1$. This implies that $N$ has a complement, which is isomorphic to $\operatorname{SL}(2,3)$. Thus it holds that $G /\left\langle a^{3^{r-3}}\right\rangle \cong C_{3^{r-3}} \times Q d(3)$ and so by a result of Loncour and Motose (see [3, page 119]), we have

$$
t\left(G /\left\langle a^{3^{r-3}}\right\rangle\right)=t\left(C_{3^{r-3}}\right)+t(\operatorname{Qd}(3))-1=3^{r-3}+8 \quad(\text { Lemma } 3) .
$$

Hence, for $r>4$, we have

$$
\begin{aligned}
t(G) & \leq t\left(\left\langle a^{3^{r-3}}\right\rangle\right) t\left(G /\left\langle a^{3^{r-3}}\right\rangle\right) \quad(\text { Wallace [3, page 313]) } \\
& =3\left(3^{r-3}+8\right)=3^{r-2}+24<3^{r-1}=3^{m-2} .
\end{aligned}
$$

We have to show that $t(G)<3^{m-2}$ for all $r \geq 4$. To this end, we need a little more calculation, and we shall give the details in [8].

LEMMA 6. If $N \cong C_{9} \times C_{9}$, then (2) holds.

Proof. Suppose $N \cong C_{9} \times C_{9}$ and set $N=\langle a, b\rangle$, where $a^{9}=b^{9}=1$. We have

$$
\mid \text { Aut } N \mid=\left(3^{4}-3^{2}\right)\left(3^{4}-3^{3}\right)=2^{4} \cdot 3^{5} \text {. }
$$

Now let $\phi \in$ Aut $N$. Then $\phi=1$ on $N / \Phi(N)$ if and only if

$$
\begin{array}{lll}
\phi(a)=a^{i} b^{j}, & \text { where } i \equiv 1, j \equiv 0 \quad(\bmod 3), \quad \text { and } \\
\phi(b)=a^{k} b^{l}, & \text { where } k \equiv 0, l \equiv 1 \quad(\bmod 3) .
\end{array}
$$


This shows that $K=\{\phi \in$ Aut $N \mid \phi=1$ on $N / \Phi(N)\}$ is a normal subgroup of Aut $N$ of order $3^{4}$. We now choose three elements $\varphi, \psi, \eta$ of Aut $N$ given by $\varphi(a)=b^{2}, \varphi(b)=a^{4} ; \quad \psi(a)=a b^{8}, \psi(b)=a^{2} b^{8} ; \quad \eta(a)=a^{4} b^{6}, \eta(b)=a^{7} b^{7}$.

Then we have $\langle\varphi, \psi\rangle \cong Q_{8}, \eta^{3}=1$; and $\eta$ acts on $\langle\varphi, \psi\rangle$ as follows:

$$
\eta^{-1} \varphi \eta=\varphi^{3} \psi, \quad \eta^{-1} \psi \eta=\varphi .
$$

We therefore see that $K:\langle\varphi, \psi, \eta\rangle$ is a (normal) subgroup of Aut $N$ of index 2. Because $O_{3,3^{\prime}, 3}(G)=G$, this shows that $G / N \cong \operatorname{SL}(2,3)$. Let $x, y, \sigma$ be the elements of $G$ corresponding to $\varphi, \psi, \eta$ respectively. Then $\langle x, y\rangle \cong Q_{8}, \sigma^{3} \in N$ and $\sigma^{-1} x \sigma=x^{3} y$, $\sigma^{-1} y \sigma=x$. Because $\sigma^{3} \in C_{N}(\langle x, y\rangle)$, we have $\sigma^{3}=1$, and so $N$ has a complement. Thus we have $G \cong N: \operatorname{SL}(2,3)$.

By using these facts, we can prove that

$$
\omega=T^{+} \alpha^{2} T^{+} \beta^{2}(f-\tau) \alpha \beta T^{+} \alpha^{2}(f-\tau) \beta^{2} T^{+} \alpha \beta(f-\tau) \alpha^{4}
$$

is a nonzero element of $J(k[G])^{27}$, where

$$
T=\left\{f, \tau, \tau^{2}\right\}, \quad f=x^{2}-1, \quad \tau=\sigma(1+x+y-x y) f, \quad \alpha=a-1, \quad \beta=b-1 .
$$

This implies $t(G)>3^{3}$, and we have $3^{3}<t(G)<3^{4}$ by Theorem 6 , Theorem 8 and Theorem 9. To prove $\omega \neq 0$, we need a little more calculation, and we shall give the details in [7].

We remark that the group $G$ discussed above satisfies the inequality $t(G)>t(P)$. This is of interest because the group is probably the first example of a $p$-solvable group satisfying this inequality. The details will be also given in [7].

LEMMA 7. If $N \cong M(3)$, then (3) holds.

Proof. Assume $N \cong M(3)$. Then $3^{2}=t(N)<t(G)$, and so $3^{2}<t(G)<3^{3}$ by Theorem 6 , Theorem 8 and Theorem 9 . Hence it suffices to prove that $G / N \cong$ $\operatorname{SL}(2,3) . N$ has a presentation:

$$
N=\left\langle a, b, c \mid a^{3}=b^{3}=c^{3}=1,[a, b]=c,[a, c]=1,[b, c]=1\right\rangle,
$$

and Aut $N$ consists of the mappings:

$$
\phi(a)=a^{i} b^{j} c^{k}, \quad \phi(b)=a^{l} b^{m} c^{n}, \quad \phi(c)=c^{s},
$$

where, $1 \leq i, j, k, l, m, n \leq 3,1 \leq s \leq 2$ and $i m-j l \equiv s(\bmod 3)$. This shows that

$$
\mid \text { Aut } M(3) \mid=2^{4} \cdot 3^{3} \text {. }
$$


Further, $K=\{\phi \in$ Aut $N \mid \phi=1$ on $N / \Phi(N)\}$ is a normal subgroup of Aut $N$ of order $3^{2}$. Now choose three elements $\varphi, \psi, \eta$ of Aut $N$ given by

$$
\begin{array}{llll}
\varphi: a \rightarrow b^{2}, & b \rightarrow a c, & c \rightarrow c, \\
\psi: a \rightarrow a b^{2} c, & b \rightarrow a^{2} b^{2}, & c \rightarrow c, \\
\eta: a \rightarrow a, & b \rightarrow a b, & c \rightarrow c .
\end{array}
$$

Then we have $\langle\varphi, \psi\rangle \cong Q_{8}, \eta^{3}=1$; and $\eta$ acts on $\langle\varphi, \psi\rangle$ as follows:

$$
\eta^{-1} \varphi \eta=\varphi^{3} \psi, \quad \eta^{-1} \psi \eta=\varphi .
$$

Thus we see that $K:\langle\varphi, \psi, \eta\rangle$ is a (normal) subgroup of Aut $N$ of index 2. This implies that $G / N \cong \mathrm{SL}(2,3)$.

REMARK 1 . We note that if $N \cong M(3)$, then $t(G)$ does not exceed 23. This fact will be used in the proof of the next lemma. We already know that $G / N \cong \operatorname{SL}(2,3)$, and hence we have $G /\langle c\rangle \cong Q d(3)$. Therefore setting

$$
A=J(k[\langle\sigma\rangle]) Q^{+} k[G], \quad B=J(k[T]) k[G], \quad C=J(k[N]) k[G],
$$

where $Q$ is a Sylow 2-subgroup of $G$ and

$$
T=\left\{f, \tau, \tau^{2}\right\}, \quad f=x^{2}-1, \quad \tau=\sigma(1+x+y-x y) f,
$$

we have

$$
J(k[G])=A+B+C \text { and }(A+B)^{5} \subset J(k[\langle c\rangle]) k[G] \text { (Lemma 3). }
$$

By the above inclusion, we have $(A+B)^{15}=0$. Therefore, because $C^{9}=0$, we obtain $J(k[G])^{23}=0$ as desired.

Lemma 8 . The case ' $N \cong M(3) \times C_{3}$ ' does not occur.

Before proving the lemma, we give the automorphism group of $M(p) \times C_{p}, p$ odd. We now set

$$
M(p)=\left\langle a, b, c \mid a^{p}=b^{p}=c^{p}=1,[a, c]=[b, c]=1,[a, b]=c\right\rangle, \quad C_{p}=\langle d\rangle .
$$

Then Aut $M(p) \times C_{p}$ consists of the mappings:

$$
\phi(a)=a^{i} b^{j} c^{k} d^{\alpha}, \quad \phi(b)=a^{l} b^{m} c^{n} d^{\beta}, \quad \phi(c)=c^{s}, \quad \phi(d)=c^{t} d^{r},
$$

where, $1 \leq i, j, k, l, m, n, t, \alpha, \beta \leq p, 1 \leq s, \gamma \leq p-1$ and $i m-j l \equiv s(\bmod p)$. This shows that

$$
\mid \text { Aut } M(p) \times C_{p} \mid=p^{6}(p+1)(p-1)^{3} .
$$

Further, a subgroup $K$ of Aut $M(p) \times C_{p}$ consisting of the mappings $\phi$ with $i=1$, $j=p, l=p, m=1, s=1, \gamma=1$ is a normal subgroup of order $p^{5}$. 
PROOF OF LEMMA 8. Assume $N \cong M(3) \times C_{3}$. Let $\varphi, \psi, \eta$ be automorphisms of $M(3) \times C_{3}$ given by

$$
\begin{array}{llll}
\varphi: a \rightarrow b^{2}, & b \rightarrow a c, & c \rightarrow c, & d \rightarrow d, \\
\psi: a \rightarrow a b^{2} c, & b \rightarrow a^{2} b^{2}, & c \rightarrow c, & d \rightarrow d, \\
\eta: a \rightarrow a, & b \rightarrow a b, & c \rightarrow c, & d \rightarrow d, \\
\rho: a \rightarrow a, & b \rightarrow b, & c \rightarrow c, & d \rightarrow d^{2} .
\end{array}
$$

Then $\langle\varphi, \psi\rangle \cong Q_{8}, \eta^{3}=1$, and $\eta$ acts on $\langle\varphi, \psi\rangle$ as follows:

$$
\eta^{-1} \varphi \eta=\varphi^{3} \psi, \quad \eta^{-1} \psi \eta=\varphi .
$$

Hence $\langle\varphi, \psi, \eta, \rho\rangle \cong \operatorname{SL}(2,3) \times C_{2}$ and $K:\langle\varphi, \psi, \eta\rangle$ is a normal subgroup of Aut $M(3) \times C_{3}$ of index 4 . This shows that $G / N \cong \operatorname{SL}(2,3)$.

Let $\sigma$ be an element of $G$ corresponding to $\eta$ and set $N=N_{1} \times N_{2}$, where $N_{1} \cong M(3), N_{2} \cong C_{3}$. Then $\sigma^{3} \in Z(N)=Z\left(N_{1}\right) \times N_{2}$. Assume first $\sigma^{3} \in Z\left(N_{1}\right)$, then $G \cong H \times C_{3}$, where $H / O_{3}(H) \cong \operatorname{SL}(2,3)$, and so

$$
t(G)=t(H)+t\left(C_{3}\right)-1 \quad(\text { Loncour, Motose [3, page 119]) }
$$

This together with Remark 1 implies that $t(G) \leq 25<3^{3}$. This contradicts our assumption.

Assume next that $\sigma^{3} \notin Z\left(N_{1}\right)$. Then to prove $t(G)<3^{3}$, we need somewhat complicated calculation, and we shall give the details in [9].

LEMMA 9. If $N \cong C_{3} \times C_{3} \times C_{3}$, then (4) or (5) holds.

PRoOF. Since Aut $C_{3} \times C_{3} \times C_{3} \cong \operatorname{GL}(3,3)$ and the maximal subgroups of $\operatorname{SL}(3,3)$ are $S_{4}, C_{13}: C_{3}$, and $\left(C_{3} \times C_{3}\right): H$ with $H / C_{2} \cong S_{4}$, we see that $G / N \cong A_{4}, C_{13}: C_{3}$ or $\operatorname{SL}(2,3)$.

If $G / N \cong C_{13}: C_{3}$, then $G$ is a group given in [4] and $t(G)=9$, which contradicts our assumption. Assume now $G / N \cong \operatorname{SL}(2,3)$. We first remark that the group generated by the following three matrices is a Sylow 2-subgroup, say $S$, of $\operatorname{SL}(3,3)$ :

$$
A=\left(\begin{array}{lll}
1 & 0 & 0 \\
0 & 0 & 2 \\
0 & 1 & 0
\end{array}\right), \quad B=\left(\begin{array}{lll}
1 & 0 & 0 \\
0 & 1 & 2 \\
0 & 2 & 2
\end{array}\right), \quad X=\left(\begin{array}{lll}
2 & 0 & 0 \\
0 & 0 & 1 \\
0 & 1 & 0
\end{array}\right),
$$

and $Q=\langle A, B\rangle$ is a unique subgroup of $S$ isomorphic to $Q_{8}$. Further,

$$
C=\left(\begin{array}{lll}
1 & 0 & 0 \\
0 & 1 & 0 \\
0 & 1 & 1
\end{array}\right) \in \operatorname{SL}(3,3)
$$


is of order 3 and normalizes $Q$. Hence $G$ is generated by $N, x, y$ and $\sigma$, where $x, y$ and $\sigma$ are elements of $G$ corresponding to $A, B$ and $C$ respectively. Clearly, we have $\langle x, y\rangle \cong Q_{8}$ and $\sigma^{3} \in N$. Now set $N=\langle a, b, c\rangle$. Then

$$
\begin{array}{lll}
a^{x}=a, & a^{y}=a, & a^{\sigma}=a, \\
b^{x}=c^{2}, & b^{y}=b c^{2}, & b^{\sigma}=b, \\
c^{x}=b, & c^{y}=b^{2} c^{2}, & c^{\sigma}=b c .
\end{array}
$$

This shows that $G /\langle a\rangle \cong Q d(3)$, and so

$$
\begin{aligned}
t(G) & \geq t(Q d(3))+t(\langle a\rangle)-1 \quad \text { (Wallace [3, page 313]) } \\
& =11>3^{2} . \quad(\text { Lemma } 3) .
\end{aligned}
$$

Thus we have $3^{2}<t(G)<3^{3}$ by Theorem 6 , Theorem 8 and Theorem 9 .

We next consider the case where $G / N \cong A_{4}$. $S$ possesses two subgroups isomorphic to $C_{2} \times C_{2}$, which are given below:

$$
T_{1}=\left\langle A^{2}, X\right\rangle, \quad T_{2}=\left\langle A^{2}, A X\right\rangle .
$$

We can choose matrices

$$
C_{1}=\left(\begin{array}{lll}
0 & 1 & 1 \\
1 & 1 & 2 \\
2 & 1 & 2
\end{array}\right), \quad C_{2}=\left(\begin{array}{lll}
0 & 0 & 1 \\
1 & 0 & 0 \\
0 & 1 & 0
\end{array}\right)
$$

of order 3 acting on $T_{1}$ and $T_{2}$ respectively. Now suppose $G / N \cong\left\langle T_{1}, C_{1}\right\rangle$ and let $x, y$ and $\sigma$ be elements of $G$ corresponding to $A^{2}, X$ and $C_{1}$ respectively. Then $\langle x, y\rangle \cong C_{2} \times C_{2}$ and $\sigma^{3} \in Z(G)=1$. This shows that $N$ has a complement and so we have $G=N:(\langle x, y\rangle:\langle\sigma\rangle)$. To complete the proof of the lemma, it suffices to prove the inequality $3^{2}<t(G)<3^{3}$. Set $U=\langle x, y\rangle$. Then, because $\langle U, \sigma\rangle\left(\cong A_{4}\right)$ is a Frobenius group, we have

$$
J(k[\langle U, \sigma\rangle])=U^{+} J(k[\langle\sigma\rangle]) \quad(\text { Wallace }[3 \text {, page 189]). }
$$

Hence

$$
J(k[G])=J(k[N]) k[G]+U^{+} J(k[\langle\sigma\rangle]) .
$$

We now set $N=\langle a, b, c\rangle$. Then

$$
Z=\left(U^{+}(\sigma-1)\right)^{2}(a-1)^{2} U^{+}(\sigma-1)
$$

is an element of $J(k[G])^{5}$. Since

$$
\begin{array}{lllll}
a^{x}=a, & a^{y}=a^{2}, & a^{\sigma}=b c, & b^{x}=b^{2}, & b^{y}=c,
\end{array}
$$


we have

$$
\begin{aligned}
Z & =U^{+} \sigma^{+}\left(a^{2}+a+1\right) U^{+}(\sigma-1)=\sigma^{+} U^{+}\left(a^{2}+a+1\right)(\sigma-1) \\
& =\sigma^{+} U^{+}\left(\left(a^{2}\right)^{\sigma}+a^{\sigma}+1\right)-\sigma^{+} U^{+}\left(a^{2}+a+1\right) \\
& =\sigma^{+} U^{+}\left((b c-1)^{2}-(a-1)^{2}\right) .
\end{aligned}
$$

Thus we have

$$
Z(a-1)^{2}(b-1)^{2}=\sigma^{+} U^{+}\langle a, b, c\rangle^{+}=G^{+}
$$

This shows that $t(G)>3^{2}$, and hence we get the inequality $3^{2}<t(G)<3^{3}$ by Theorem 6, Theorem 8 and Theorem 9 as desired. If $G / N \cong\left\langle T_{2}, C_{2}\right\rangle$ then we can prove that $G \cong\left(C_{3} \times C_{3} \times C_{3}\right): A_{4}$ and $3^{2}<t(G)<3^{3}$ by the same argument as the above.

REMARK 2. We note here that if $G / N \cong\left\langle T_{2}, C_{2}\right\rangle$, then $G=\langle N, x, y, \sigma\rangle$, where $\langle x, y\rangle \cong C_{2} \times C_{2}$ and $\sigma^{3}=1$, and

$$
\begin{aligned}
& a^{x}=a, \quad a^{y}=a^{2}, \quad a^{\sigma}=c, \quad b^{x}=b^{2}, \quad b^{y}=b^{2}, \quad b^{\sigma}=a, \\
& c^{x}=c^{2}, \quad c^{y}=c, \quad c^{\sigma}=b, \quad x^{\sigma}=y, \quad y^{\sigma}=x y .
\end{aligned}
$$

These equalities will be used in the proof of the next lemma.

LEMMA 10. If $N \cong C_{3^{m-3}} \times C_{3} \times C_{3}$ with $m \geq 5$, then (6) holds.

Proof. Assume $N \cong C_{3^{m-3}} \times C_{3} \times C_{3}$. Then $G / N$ is contained isomorphically in $\mathrm{GL}(3,3)$, and so $G / N \cong A_{4}, C_{13}: C_{3}$ or $\operatorname{SL}(2,3)$.

If $G / N \cong C_{13}: C_{3}$ then

$$
\begin{aligned}
t(G) & \leq t(\Phi(N)) \cdot t(G / \Phi(N)) \quad \text { (Wallace [3, page 313]) } \\
& =3^{m-4} \cdot 3^{2} \quad(\text { Motose [4]) } \\
& =3^{m-2}
\end{aligned}
$$

which contradicts our assumption.

Next we show that the case ' $G / N \cong A_{4}$ ' also does not occur. Set $N=\langle a, b, c\rangle$, where $a^{3^{m-3}}=b^{3}=c^{3}=1$. Let $\langle x, y\rangle\left(\cong C_{2} \times C_{2}\right)$ be a Sylow 2-subgroup of $G$ and $\sigma$ an element of $G-(N:\langle x, y\rangle)$ such that $\sigma^{3} \in N$. If $G /\left\langle a^{3}\right\rangle$ is a group discussed in the proof of Lemma 9, then one of the following holds:

$$
a^{\sigma} \equiv b c, \quad b^{\sigma} \equiv a c, \quad c^{\sigma} \equiv a b \quad\left(\bmod \left\langle a^{3}\right\rangle\right)
$$

But this is impossible because if $a^{\sigma} \equiv b c$ then the order of $b c$ would be $3^{m-3}$, and the other two cases are also impossible. If $G /\left\langle a^{3}\right\rangle$ is a group given in Remark 2 , we also reach a contradiction by using the equalities given there. 
Assume now $G / N \cong \operatorname{SL}(2,3)$. Let $Q=\langle x, y\rangle\left(\cong Q_{8}\right)$ be a Sylow 2-subgroup of $G$ and $\sigma$ an element of $G-(N:\langle x, y\rangle)$ such that $\sigma^{3} \in N$. Then $G$ is generated by $a, b, c$ and $\sigma$. In view of the proof of Lemma 9, we may assume that the the following holds:

$$
\begin{array}{lll}
a^{x}=a^{1+3 i}, & a^{y}=a^{1+3 j}, & a^{\sigma}=a^{1+3 k}, \\
b^{x}=a^{3^{m-4} \alpha} c^{2}, & b^{y}=a^{3^{m-4} \beta} b c^{2}, & b^{\sigma}=a^{3^{m-4} \gamma} b, \\
c^{x}=a^{3^{m-4} \lambda} b, & c^{y}=a^{3^{m-4} \mu} b^{2} c^{2}, & c^{\sigma}=a^{3^{m-4} v} b c,
\end{array}
$$

where $0 \leq i, j, k \leq 3^{m-3}-1,0 \leq \alpha, \beta, \gamma, \lambda, \mu, \nu \leq 2$. Because $x^{4}=y^{4}=1$, the first two equalities force $i$ and $j$ to be 0 . This implies that $Z(N Q)=\langle a\rangle$, and so $\sigma^{3} \in\langle a\rangle$.

We now show that $\sigma^{3} \notin\left\langle a^{3}\right\rangle$. By way of contradiction, we assume $\sigma^{3} \in\left\langle a^{3}\right\rangle$. Then $\langle a, \sigma\rangle$ is a 3 -group of order $3^{m-2}$ and exponent $3^{m-3}$, and so $\langle a\rangle$ has a complement in $\langle a, \sigma\rangle$. Thus we may assume that $\sigma^{3}=1$. We now show that the inequality $t(G) \leq 3^{m-2}$ holds, which contradicts our assumption. By Lemma 3, we have

$$
J(k[G])=J(k[\langle\sigma\rangle]) Q^{+} k[G]+J(k[T]) k[G]+J(k[N]) k[G],
$$

where

$$
T=\left\{f, \tau, \tau^{2}\right\}, \quad f=x^{2}-1, \quad \tau=\sigma(1+x+y-x y) f .
$$

Now set $\bar{G}=G /\left\langle a^{3}\right\rangle$. Then $\bar{G} \cong C_{3} \times Q d(3)$, and

$$
J(k[\bar{G}])=J(k[\langle\bar{\sigma}\rangle]) \bar{Q}^{+} k[\bar{G}]+J(k[\bar{T}]) k[\bar{G}]+J(k[\bar{N}]) k[\bar{G}] .
$$

Because

$$
\left(J(k[\langle\bar{\sigma}\rangle]) \bar{Q}^{+} k[\bar{G}]+J(k[\bar{T}]) k[\bar{G}]\right)^{5}=0 \quad(\text { Lemma 3) }
$$

we have

$$
\left(J(k[\langle\sigma\rangle]) Q^{+} k[G]+J(k[T]) k[G]\right)^{5} \subset J\left(k\left[\left\langle a^{3}\right\rangle\right]\right) k[G],
$$

and so

$$
\left(J(k[\langle\sigma\rangle]) Q^{+} k[G]+J(k[T]) k[G]\right)^{5 \cdot 3^{m-4}}=0 .
$$

On the other hand, by a result of Loncour and Motose (see [3, page 119]) we have

$$
(J(k[N]) k[G])^{3^{m-3}+4}=J(k[N])^{3^{m-3}+4} k[G]=0 .
$$

We therefore get

$$
J(k[G])^{3^{m-3}+5 \cdot 3^{m-4}+3}=0
$$

and $t(G) \leq 3^{m-2}$ as desired.

Thus we have $\sigma^{3} \notin\left\langle a^{3}\right\rangle$. To complete the proof, it suffices to prove that the inequality $3^{m-2}<t(G)<3^{m-1}$ holds in this case. We may assume that $\sigma^{3}=a$, from 
which it follows that $k=0$. Because $x^{2}=y^{2}$, we have $b^{x^{2}}=b^{y^{2}}$ and $c^{x^{2}}=c^{y^{2}}$. These equalities give the following congruences:

$$
\alpha+2 \lambda \equiv 2 \beta+2 \mu, \quad \alpha+\lambda \equiv 2 \beta \quad(\bmod 3) .
$$

Similarly, from the equalities $x^{y}=x^{3}, x^{\sigma}=x^{3} y, y^{\sigma}=y$ the following congruences follows:

$$
\begin{array}{llr}
2 \alpha+2 \beta+\lambda \equiv 2 \lambda, & \alpha+\lambda+\mu \equiv \alpha & (\bmod 3), \\
\alpha+2 \gamma+2 v \equiv 2 \lambda+\mu, & 2 \alpha+2 \gamma+\lambda \equiv \alpha+2 \beta & (\bmod 3), \\
\beta+2 \nu \equiv \alpha, & 2 \beta+2 \gamma+\mu+2 v \equiv \lambda & (\bmod 3)
\end{array}
$$

These congruences with respect to $(\alpha, \beta, \gamma, \lambda, \mu, \nu)$ have the following solutions:
(i) $(0,0,0,0,0,0)$
(ii) $(1,2,0,0,0,1$,
(iii) $(2,1,0,0,0,2)$
(iv) $(1,1,0,1,2,0)$
(v) $(2,2,0,2,1,0)$
(vi) $(2,0,0,1,2,1)$
(vii) $(1,0,0,2,1,2)$
(viii)
$(0,2,0,1,2,2)$
(ix) $(0,1,0,2,1,1)$.

If (i) holds, then we have

$$
\left\{\begin{array}{lll}
a^{x}=a, & a^{y}=a, & a^{\sigma}=a, \\
b^{x}=c^{2}, & b^{y}=b c^{2}, & b^{\sigma}=b, \\
c^{x}=b, & c^{y}=b^{2} c^{2}, & c^{\sigma}=b c .
\end{array}\right.
$$

If (ii) holds, then setting $B=a^{3^{m-4}} b, C=a^{3^{m-4}} c$, we have

$$
B^{x}=C^{2}, \quad B^{y}=B C^{2}, \quad B^{\sigma}=B, \quad C^{x}=B, \quad C^{y}=B^{2} C^{2}, \quad C^{\sigma}=B C .
$$

This shows that the group corresponding to the solution (ii) is isomorphic to the one corresponding to the solution (i). One can also prove that the groups corresponding to the solutions (iii)-(ix) are all isomorphic to the one corresponding to the solution (i). Therefore, $G$ is generated by $N, x, y$ and $\sigma$ and the action of $x, y$ and $\sigma$ on $N$ is given by $(*)$. Set

$$
Z=J(k[\langle\sigma\rangle]) Q^{+} k[G]
$$

Then $Z \subset J(k[G])$ (Lemma 3$)$ and

$$
\begin{aligned}
Z^{2} & =J(k[\langle\sigma\rangle]) Q^{+} k[N] J(k[\langle\sigma\rangle]) Q^{+} k[G] \\
& =J(k[\langle\sigma\rangle]) \cdot Q^{+} k[N] Q^{+} \cdot J(k[\langle\sigma\rangle]) k[G] .
\end{aligned}
$$

Since $Q^{+} k[N] Q^{+}$is a $k$-space generated by the elements of the form $Q^{+} \sum_{g \in Q} u^{g}$ $(u \in N)$, we see that $\sigma$ acts trivially on $Q^{+} k[N] Q^{+}$. Hence

$$
Z^{2}=J(k[\langle\sigma\rangle])^{2} Q^{+} k[N] Q^{+} k[G],
$$


and we have

$$
Z^{i}=J(k[\langle\sigma\rangle])^{i} Q^{+} k[N] Q^{+} k[G]
$$

for every $i$. We therefore see that the nilpotency index of $Z$ is $3^{m-2}$, and $\operatorname{sot}(G)>3^{m-2}$. This together with Theorem 6 , Theorem 8 and Theorem 9 implies $3^{m-2}<t(G)<3^{m-1}$ as desired.

\section{References}

[1] J. Conway, R. Curtis, S. Norton, R. Parker and R. Wilson, Atlas of finite groups (Clarendon Press, Oxford. Amsterdam, 1985).

[2] P. Hall and G. Higman, 'On the $p$-length of $p$-solvable groups and reduction theorems for Burnside's problem', Proc. London Math. Soc. (3) 6 (1956), 1-42.

[3] G. Karpilovsky, The Jacobson radical of group algebras (North-Holland, Amsterdam, 1987).

[4] K. Motose, 'On the nilpotency index of the radical of a group algebra, III', J. London Math. Soc. (2) 25 (1982), 39-42.

[5] Y. Ninomiya, 'Finite $p$-groups with cyclic subgroups of index $p^{2}$, Math. J. Okayama Univ. 36 (1994), 1-21.

[6] — - 'Nilpotency indices of the radicals of p-group algebras', Proc. Edinburgh Math. Soc. 37 (1994). 509-517.

[7] _ - 'Nilpotency indices of the radicals of finite $p$-solvable group algebras, II', Comm. Algebra, to appear.

[8] - "Nilpotency indices of the radicals of finite $p$-solvable group algebras, III', preprint, 1999.

[9] _ N . Nilpotency indices of the radicals of finite $p$-solvable group algebras, IV', preprint, 1999.

[10] A. Shalev. 'Dimension subgroups, nilpotency indices, and the number of generators of ideals in p-group algebras', I. Algebra 129 (1990), 412-438.

Department of Mathematical Sciences

Faculty of Science

Shinshu University

Matsumoto 390-8621

Japan

e-mail: ysninom@gipac.shinshu-u.ac.jp 